\title{
EXACT-APPROXIMATE BOUNDARY CONTROLLABILITY OF THERMOELASTIC SYSTEMS UNDER FREE BOUNDARY CONDITIONS
}

\author{
George Avalos \\ Department of Mathematics and Statistics \\ Texas Tech University \\ Lubbock, Texas 79409 USA \\ email: avalos@math.ttu.edu \\ Irena Lasiecka \\ Department of Applied Mathematics \\ University of Virginia \\ Charlottesville, Virginia 22903 USA \\ email: il2v@amsun.apma.virginia.edu
}

\begin{abstract}
Controllability properties of a partial differential equation (PDE) model describing a thermoelastic plate are studied. The PDE is comprised of a Kirchoff plate equation coupled to a heat equation on a bounded domain, with the coupling taking place on the interior and boundary of the domain. The coupling in this PDE is parameterized by $\alpha>0$. Control is exerted through the (two) free boundary conditions of the plate equation, and through the Robin boundary condition of the temperature. These controls have the physical interpretation, respectively, of inserted forces and moments, and prescribed temperature, all of which act on the edges of the plate. The main result here is that under such boundary control, and with initial data in the basic space of wellposedness, one can simultaneously control the displacement of the plate $e x$ actly, and the temperature approximately. Moreover, the thermal control may be taken to be arbitrarily smooth in time and space, and the thermal control region may be any nonempty subset of the boundary. This controllability holds for arbitrary values of the coupling parameter $\alpha$.
\end{abstract}

The original version of this chapter was revised: The copyright line was incorrect. This has been corrected. The Erratum to this chapter is available at DOI: 10.1007/978-0-387-35359-3_40 


\section{INTRODUCTION}

\section{Statement of the Problem}

Let $\Omega$ be a bounded open subset of $\mathbb{R}^{2}$ with its sufficiently smooth boundary denoted as $\Gamma$. The boundary will be decomposed as $\Gamma=\overline{\Gamma_{0}} \cup \overline{\Gamma_{1}}$, with both $\Gamma_{0}$ and $\Gamma_{1}$ being open and nonempty, and further satisfying $\overline{\Gamma_{0}} \cap \overline{\Gamma_{1}}=\varnothing$. In addition, let $\Gamma_{2}$ be any open and nonempty subset of $\Gamma$. With this geometry, we shall consider here the following thermoelastic system on finite time $(0, T)$ :

$$
\left\{\begin{array}{l}
\left\{\begin{array}{l}
\omega_{t t}-\gamma \Delta \omega_{t t}+\Delta^{2} \omega+\alpha \Delta \theta=0 \\
\beta \theta_{t}-\eta \Delta \theta+\sigma \theta-\alpha \Delta \omega_{t}=0
\end{array} \text { on }(0, T) \times \Omega ;\right. \\
\omega=\frac{\partial \omega}{\partial \nu}=0 \text { on }(0, T) \times \Gamma_{0} \\
\left\{\begin{array}{l}
\Delta \omega+(1-\mu) B_{1} \omega+\alpha \theta=u_{1} \\
\frac{\partial \Delta \omega}{\partial \nu}+(1-\mu) \frac{\partial B_{2} \omega}{\partial \tau}-\gamma \frac{\partial \omega_{t t}}{\partial \nu}+\alpha \frac{\partial \theta}{\partial \nu}=u_{2}
\end{array} \text { on }(0, T) \times \Gamma_{1}\right. \\
\frac{\partial \theta}{\partial \nu}+\lambda \theta=\left\{\begin{array}{cc}
u_{3} & \text { on }(0, T) \times \Gamma_{2} \\
0 & \text { on }(0, T) \times \Gamma \backslash \Gamma_{2}
\end{array} \quad \lambda \geq 0\right. \\
\omega(t=0)=\omega_{0}, \omega_{t}(t=0)=\omega_{1}, \theta(t=0)=\theta_{0} \text { on } \Omega .
\end{array}\right.
$$

Here, $\alpha, \beta, \eta$ and $\sigma$ are positive parameters. The positive constant $\gamma$ is proportional to the thickness of the plate and assumed to be small with $0<\gamma \leq M$. The boundary operators $B_{i}$ are given by $B_{1} \omega \equiv 2 \nu_{1} \nu_{2} \frac{\partial^{2} \omega}{\partial x \partial y}-\nu_{1}^{2} \frac{\partial^{2} \omega}{\partial y^{2}}-\nu_{2}^{2} \frac{\partial^{2} \omega}{\partial x^{2}}$, and $B_{2} \omega \equiv\left(\nu_{1}^{2}-\nu_{2}^{2}\right) \frac{\partial^{2} \omega}{\partial x \partial y}+\nu_{1} \nu_{2}\left(\frac{\partial^{2} \omega}{\partial y^{2}}-\frac{\partial^{2} \omega}{\partial x^{2}}\right)$. The constant $\mu \in\left(0, \frac{1}{2}\right)$ is the familiar Poisson's ratio, and $\nu=\left[\nu_{1}, \nu_{2}\right]$ denotes the outward unit normal to the boundary. Here we shall also make the following geometric assumption on the (uncontrolled) portion of the boundary $\Gamma_{0}$ :

$$
\exists\left\{x_{0}, y_{0}\right\} \in \mathbb{R}^{2} \text { such that } \bar{h}(x, y) \cdot \nu \leq 0 \text { on } \Gamma_{0},
$$

where $\bar{h}(x, y) \equiv\left[x-x_{0}, y-y_{0}\right]$.

The PDE model (1.1), with boundary functions $u_{1}=u_{2}=0$, and $u_{3}=0$, mathematically describes an uncontrolled Kirchoff plate subjected to a thermal damping, with the displacement of the plate represented by the function $\omega(t, x, y)$, and the temperature given by the function $\theta(t, x, y)$ (see [8]). The given control variables $u_{1}(t, x)$ and $u_{2}(t, x)$ are defined on the portion of the boundary $(0, T) \times \Gamma_{1}$; the control $u_{3}(t, x)$ is defined on $(0, T) \times \Gamma_{2}$.

With the denotation

$$
H_{\Gamma_{0}}^{k}(\Omega) \equiv\left\{\varpi \in H^{k}(\Omega):\left.\frac{\partial^{j} \varpi}{\partial \nu^{j}}\right|_{\Gamma_{0}}=0 \text { for } j=0, \ldots, k-1\right\}
$$


we will throughout take the initial data $\left[\omega_{0}, \omega_{1}, \theta_{0}\right]$ to be in $H_{\Gamma_{0}}^{2}(\Omega)^{\circ} \times H_{\Gamma_{0}}^{1}(\Omega) \times$ $L^{2}(\Omega)$. In this paper, we will study controllability properties of solutions of (1.1) under the influence of boundary control functions in preassigned spaces. In particular we intend to address, on the finite time interval $[0, T]$, the following problem of exact-approximate controllability with respect to the basic energy space $H_{\Gamma_{0}}^{2}(\Omega) \times H_{\Gamma_{0}}^{1}(\Omega) \times L^{2}(\Omega)$ (this term being coined in [5]): For given data $\left[\omega_{0}, \omega_{1}, \theta_{0}\right]$ (initial) and $\left[\omega_{0}^{T}, \omega_{1}^{T}, \theta_{0}^{T}\right]$ (terminal) in $H_{\Gamma_{0}}^{2}(\Omega) \times H_{\Gamma_{0}}^{1}(\Omega) \times L^{2}(\Omega)$ and arbitrary $\epsilon>0$, we seek a suitable control triple $\left[u_{1}, u_{2}, u_{3}\right] \in L^{2}\left(0, T ; L^{2}\left(\Gamma_{1}\right) \times\right.$ $\left.H^{-1}\left(\Gamma_{1}\right)\right) \times C^{r}\left(\Sigma_{2, T}\right)$ (where $r>0$ and $\left.\Sigma_{i, T}=(0, T) \times \Gamma_{i}, i=0,1,2\right)$ such that the corresponding solution $\left[\omega, \omega_{t}, \theta\right]$ of $(1.1)$ satisfies the steering property at terminal time $T$

$$
\left[\omega(T), \omega_{t}(T)\right]=\left[\omega_{0}^{T}, \omega_{1}^{T}\right] ; \text { and }\left\|\theta(T)-\theta_{0}^{T}\right\|_{L^{2}(\Omega)} \leq \epsilon
$$

In regards to the literature on this particular problem, the most relevant work is that of J. Lagnese in [9]. Therein, Lagnese shows that if the coupling parameter $\alpha$ is small enough and the boundary $\Gamma$ is "star-shaped", then the boundary-controlled system (1.1) is (partially) exactly controllable with respect to the displacement $\omega$. Also in [14], a boundary-controlled thermoelastic wave equation is studied, with a coupling parameter $\alpha$ likewise present therein, and a result of partial exact controllability (again for the displacement) for this PDE is cited. This controllability result is quoted to be valid for all sizes of $\alpha$; however in [15], the author has acknowledged a flaw in the controllability proof, the correction of which necessitates a smallness criterion on $\alpha$, akin to the situation in [9]. The chief contribution of the present paper is to remove restrictions on the size of the coupling parameter (see Theorem 3 below), at the expense of adding the arbitrarily smooth boundary control $u_{3}$ in the thermal component. For a 1-D version of (1.1), S. Hansen and B. Zhang in [6], via a moment problem approach, show the system's exact null controllability with boundary control in either the plate or thermal component. Other controllability results for the thermoelastic system which do not assume any "smallness" condition on the coupling parameters deal with distributed/internal controls. Such include that in [5], in which interior control is placed in the Kirchoff plate component subject to clamped boundary conditions; with such control, one obtains exact controllability for the displacement $\omega$, and approximate controllability for the temperature $\theta$. Alternatively in [3], interior control is placed in the heat equation of (1.1) so as to obtain exact controllability for both components $\omega$ and $\theta$. In addition, the work in [13] deals with obtaining a result of null controllability for both components of a coupled wave and heat equation, in the case that interior control is inserted in the wave component only.

So again, the main contribution and novelty of this paper is that we consider boundary controls acting via the higher order free boundary conditions, and we do not assume any size restriction on the coupling parameter $\alpha$. Moreover, we do not impose any geometric "star-shaped" conditions on the controlled portion of the geometry. 
It should be noted that the particular type of boundary conditions imposed on the mechanical variables greatly affects the analysis of the problem, even in the case of internal control. Indeed, in the case of all boundary conditions, save for the free case, it is known that the thermoelastic plate semigroup can be decomposed into a damped Kirchoff plate semigroup and a compact perturbation (see [5] and [12]). Since controllability estimates are invariant with respect to compact perturbations (at least in the case of approximately controllable systems, which we are dealing with here), the aforesaid decomposition, valid for the case of lower order boundary conditions, reduces the problem of exact controllability for the mechanical variable to that of uncoupled Kirchoff plates. Thus, the case of lower order boundary conditions allows a reduction of the coupled problem into one which has been much studied in the past. This strategy, while successfully employed in the case of clamped or hinged boundary conditions (see [5]), is not applicable here. Indeed, in our present case of free boundary conditions, there is no decomposition with a compact part, as in the lower order case (see [12]); moreover, the controllability operator corresponding to the given boundary controls is not bounded on the natural energy space. This latter complication is due to the fact that the Lopatinski conditions are not satisfied for the Kirchoff model under free boundary conditions.

The strategy adopted in this paper consists of the following steps: Initially, a suitable transformation of variables is made and applied to the equation (1.1); subsequently, a multiplier method is invoked with respect to the transformed equation. The mulitiplers employed here are the differential multipliers used in the study of exact controllability for the Kirchoff plate model, together with the nonlocal ( $\Psi D O)$ multipliers used in the study of thermoelastic plates in [1] and [2]. This multiplier method allows the attainment of preliminary estimates for the energy of the system. However, these estimates are "polluted" by certain boundary terms which are not majorized by the energy. To cope with these, we use the sharp trace estimates established in [11] for Kirchoff plates. The use of this PDE result introduces lower order terms into the energy estimate, which are eventually eliminated with the help of a new unique continuation result in [7]. It is only at the level of invoking this uniqueness result that the thermal control $u_{3}$ on $\Gamma_{2}$ must be introduced.

We post our main result here on controllability.

Theorem 1 Let the assumption (1.2) stand. There is then a $T^{*}>0$ so that for $T>T^{*}$ the following controllability property holds true: For given initial data $\left[\omega_{0}, \omega_{1}, \theta_{0}\right]$ and terminal data $\left[\omega_{0}^{T}, \omega_{1}^{T}, \theta_{0}^{T}\right]$ in the space $H_{\Gamma_{0}}^{2}(\Omega) \times H_{\Gamma_{0}}^{1}(\Omega) \times$ $L^{2}(\Omega)$, and arbitrary $\epsilon>0$, one can find control functions $\left[u_{1}^{*}, u_{2}^{*}, u_{3}^{*}\right] \in$ $L^{2}\left(0, T ; L^{2}\left(\Gamma_{1}\right) \times H^{-1}\left(\Gamma_{1}\right)\right) \times C^{r}\left(\Sigma_{2, T}\right)$ (where given $\left.r>0\right)$ such that the corresponding solution $\left[\omega^{*}, \omega_{t}^{*}, \theta^{*}\right]$ to (1.1) satisfies (1.3) at terminal time $T$.

Remark 2 The presence of the control $u_{3}$ in Theorem 1 is owing solely to the need to invoke the aforementioned uniqueness result of Isakov in the proof below; it plays no part whatsoever in obtaining the preliminary (lower order term-tainted) estimate on the energy. Consequently, we have the freedom to 
prescribe the thermal control region to be as small as we wish, and the control $u_{3}$ to be as smooth in time and space as desired.

\section{PROOF OF THEOREM 1}

A preponderant portion of the proof of Theorem 1 is wrapped up in showing the following result of exact controllability for the displacement only:

Theorem 3 With the coupling parameter $\alpha$ in (1.1) being arbitrary and the assumption (1.2) in place, there is then a $T^{*}>0$ so that for $T>T^{*}$, the following property holds true: For all initial data $\left[\omega_{0}, \omega_{1}, \theta_{0}\right] \in H_{\Gamma_{0}}^{2}(\Omega) \times H_{\Gamma_{0}}^{1}(\Omega) \times L^{2}(\Omega)$ and terminal data $\left[\omega_{0}^{T}, \omega_{1}^{T}\right] \in H_{\Gamma_{0}}^{2}(\Omega) \times H_{\Gamma_{0}}^{1}(\Omega)$, there exists $\left[u_{1}, u_{2}, u_{3}\right] \in$ $L^{2}\left(0, T ; L^{2}\left(\Gamma_{1}\right) \times H^{-1}\left(\Gamma_{1}\right)\right) \times H^{s}\left(\Sigma_{2, T}\right)$, where arbitrary $s \geq 0$, such that the corresponding solution $\left[\omega, \omega_{t}, \theta\right]$ to (1.1) satisfies $\left[\omega(T), \omega_{t}(T)\right]=\left[\omega_{0}^{T}, \omega_{1}^{T}\right]$.

Indeed, if Theorem 3 is shown to be true, then using the miminal norm steering control (see Appendix B of [10]), one can, in a straightforward fashion, construct a control $\left[u_{1}^{*}, u_{2}^{*}, u_{3}^{*}\right]$ such that the corresponding trajectory $\left[\omega^{*}, \omega_{t}^{*}, \theta^{*}\right]$ has the desired reachability property (1.3). (See [4] for the precise details). Accordingly, the sequel is devoted to showing the validity of Theorem 3.

The theme of the proof of Theorem 3 is a classical one. Denoting the control space $\mathcal{U}_{s} \equiv L^{2}\left(\Gamma_{1}\right) \times H^{-1}\left(\Gamma_{1}\right) \times H^{s}\left(\Gamma_{2}\right)$, one defines the operator $\mathcal{L}_{T}$ : $D\left(\mathcal{L}_{T}\right) \subset \mathcal{U} \rightarrow H_{\Gamma_{0}}^{2}(\Omega) \times H_{\Gamma_{0}}^{1}(\Omega)$ to be that which takes the terminal control to the terminal state; i.e., $\mathcal{L}_{T}\left[u_{1}, u_{2}, u_{3}\right]=\left[\omega(T), \omega_{t}(T)\right]$, where $\left[\omega(T), \omega_{t}(T)\right]$ is the plate component of the solution to (1.1) at time $t=T$. As it is defined, $\mathcal{L}_{T}$ is a closed, unbounded operator, with its domain being densely defined. By a principle of functional analysis then (see e.g., [16]), to prove Theorem 3 , which is essentially a statement of the surjectivity of $\mathcal{L}_{T}$, it is enough to establish the PDE inequality

$$
\int_{0}^{T}\left\|\left|\nabla \widehat{\phi}_{t}\right|\right\|_{L^{2}\left(\Gamma_{1}\right)}^{2} d t+\|\psi\|_{\left[H^{s}\left(\Sigma_{2, T}\right)\right]^{\prime}}^{2} \geq C_{T}\left\|\left[\phi_{0}, \phi_{1}\right]\right\|_{H_{\Gamma_{0}}^{2}(\Omega) \times H_{\Gamma_{0}}^{1}(\Omega)}^{2}
$$

where $\left[\phi, \phi_{t}, \psi\right]$ is the solution to the following backwards system, corresponding to terminal data $\left[\phi_{0}, \phi_{1}\right] \in D\left(\mathcal{L}_{T}^{*}\right)$ : 


$$
\left\{\begin{array}{l}
\left\{\begin{array}{l}
\phi_{t t}-\gamma \Delta \phi_{t t}+\Delta^{2} \phi+\alpha \Delta \psi=0 \\
\beta \psi_{t}+\eta \Delta \psi-\sigma \psi-\alpha \Delta \phi_{t}=0
\end{array} \text { on }(0, T) \times \Omega\right. \\
\phi=\frac{\partial \phi}{\partial \nu}=0 \text { on } \Sigma_{0, T} \\
\left\{\begin{array}{l}
\Delta \phi+(1-\mu) B_{1} \phi+\alpha \psi=0 \\
\frac{\partial \Delta \phi}{\partial \nu}+(1-\mu) \frac{\partial B_{2} \phi}{\partial \tau}-\gamma \frac{\partial \phi_{t t}}{\partial \nu}+\alpha \frac{\partial \psi}{\partial \nu}=0
\end{array} \text { on } \Sigma_{1, T}\right. \\
\frac{\partial \psi}{\partial \nu}+\lambda \psi=0 \quad \text { on }(0, T) \times \Gamma, \lambda \geq 0 \\
{\left[\phi(T), \phi_{t}(T), \psi(T)\right]=\left[\phi_{0}, \phi_{1}, 0\right]}
\end{array}\right.
$$

The proof of Theorem 3 is then based upon showing the inequality (2.1), at least for $T>0$ large enough, where $\left[\left.\frac{\partial \phi_{t}}{\partial \nu}\right|_{\Gamma_{1}},\left.\phi_{t}\right|_{\Gamma_{1}},\left.\psi\right|_{\Gamma_{2}}\right]$ are traces of the solution $\left[\phi, \phi_{t}, \psi\right]$ to the backwards system (2.2). Because of space constraints, we give here only a broad sketch of the proof of Theorem 3; the full particulars are provided in [4].

Step 1. We start by making the substitution

$$
\widehat{\phi}(t)=e^{-\xi t} \phi(t) ; \text { and } \widehat{\psi}(t)=e^{-\xi t} \psi(t)
$$

where parameter $\xi \equiv \frac{\alpha^{2}}{2 \gamma \eta}$. This particular choice of parameter allows the PDE (2.2) to be transformed into the following plate equation whose forcing function is comprised in part of the high order term $\widehat{\psi}_{t}$ :

$$
\left\{\begin{array}{l}
\widehat{\phi}_{t t}-\gamma \Delta \widehat{\phi}_{t t}+\Delta^{2} \widehat{\phi}=c_{0} \widehat{\psi}+c_{1} \widehat{\psi}_{t}+c_{2} \widehat{\phi}+c_{3} \widehat{\phi}_{t}+c_{4} \Delta \widehat{\phi} \quad \text { on }(0, T) \times \Omega ; \\
\widehat{\phi}=\frac{\partial \widehat{\phi}}{\partial \nu}=0 \text { on } \Sigma_{0, T} \\
\left\{\begin{array}{l}
\Delta \widehat{\phi}+(1-\mu) B_{1} \widehat{\phi}=-\alpha \widehat{\psi} \\
\frac{\partial \Delta \hat{\phi}}{\partial \nu}+(1-\mu) \frac{\partial B_{2} \widehat{\phi}}{\partial \tau}=\gamma \frac{\partial}{\partial \nu}\left(\xi^{2} \widehat{\phi}+2 \xi \widehat{\phi}_{t}+\widehat{\phi}_{t t}\right)-\alpha \frac{\partial \widehat{\psi}}{\partial \nu} \quad \text { on } \Sigma_{1, T} ; \\
{\left[\widehat{\phi}(T), \widehat{\phi}_{t}(T), \widehat{\psi}(T)\right]=\left[e^{-\xi T} \phi_{0},-\xi e^{-\xi T} \phi_{0}+e^{-\xi T} \phi_{1}, 0\right]}
\end{array}\right.
\end{array}\right.
$$

where the $c_{i}$ are constants depending on the physical parameters.

Step 2. At this point we invoke a multiplier method with respect to the uncoupled equation in (2.4), using two multipliers which are, respectively, standard (as in [9]) and nonstandard (as in [1] and [2]). To wit, we multiply this 
equation by $\widehat{\phi}_{t}$, and subsequently integrate in time and space so as to arrive at

$$
\begin{aligned}
& \frac{1}{2}\left[\|\widehat{\phi}(t)\|_{H_{\Gamma_{0}}^{2}(\Omega)}^{2}+\left\|\widehat{\phi}_{t}(t)\right\|_{H_{\Gamma_{0}}^{1}(\Omega)}^{2}\right]_{t=s}^{t=\tau}=-\alpha \int_{s}^{\tau}\left(\widehat{\psi}, \frac{\partial \widehat{\phi}_{t}}{\partial \nu}\right)_{L^{2}\left(\Gamma_{1}\right)} d t \\
& -\int_{s}^{\tau}\left(\gamma \xi^{2} \frac{\partial \widehat{\phi}}{\partial \nu}+2 \gamma \xi \frac{\partial \widehat{\phi}_{t}}{\partial \nu}+\alpha \lambda \gamma_{0} \widehat{\psi}, \widehat{\phi}_{t}\right)_{L^{2}\left(\Gamma_{1}\right)} d t \\
& +\int_{s}^{\tau}\left(c_{0} \widehat{\psi}+c_{2} \widehat{\phi}+c_{3} \widehat{\phi}_{t}+c_{4} \Delta \widehat{\phi}+c_{1} \widehat{\psi}_{t}, \widehat{\phi}_{t}\right)_{L^{2}\left(\Gamma_{1}\right)} d t
\end{aligned}
$$

Moreover, letting $A_{D}$ denote the Laplacian operator with Dirichlet boundary conditions, and $A_{D}^{-1} \in \mathcal{L}\left(L^{2}(\Omega)\right)$ its corresponding (smoothing) inverse, we then multiply the PDE in (2.4) by $-\frac{c_{1}}{\gamma} A_{D}^{-1} \widehat{\psi}$, and thereafter integrate in time and space. Adding the resultant expression to (2.5), and subsequently majorizing the sum, we obtain the following:

Lemma 4 The solution $\left[\widehat{\phi}, \widehat{\phi}_{t}, \widehat{\psi}\right]$ to (2.4) satisfies the following relation for all $s$ and $\tau \in[0, T]$ :

$$
\begin{aligned}
& {\left[\|\hat{\phi}(t)\|_{H_{\Gamma_{0}}^{2}(\Omega)}^{2}+\left\|\widehat{\phi}_{t}(t)\right\|_{H_{\Gamma_{0}}^{1}(\Omega)}^{2}\right]_{t=s}^{t=\tau} } \\
\leq & C \int_{0}^{T}\left\|\left|\nabla \widehat{\phi}_{t}\right|\right\|_{L^{2}\left(\Gamma_{1}\right)}^{2} d t+\text { I.o.t. }\left(\widehat{\psi}, \widehat{\phi}, \widehat{\phi}_{t}\right),
\end{aligned}
$$

where l.o.t $\left(\widehat{\psi}, \widehat{\phi}, \widehat{\phi}_{t}\right)$ denotes, as usual, "lower order terms" (below the energy level) of $\widehat{\psi}, \hat{\phi}$, and $\hat{\phi}_{t}$.

Step 3. Taking a radial vector field $\bar{h} \in \mathbb{R}^{2}$ which meets the requirement in (1.2), one can derive the following inequality which is an analogue to that demonstrated in [9]. In deriving this estimate the trace result in [11] is critically invoked.

Lemma 5 For all $\epsilon_{0} \in(0, T)$, the solution $\left[\widehat{\phi}, \widehat{\phi}_{t}\right]$ to (2.4) satisfies

$$
\begin{aligned}
& \int_{\epsilon_{0}}^{T-\epsilon_{0}}\left[\|\hat{\phi}\|_{H_{\Gamma_{0}}^{2}(\Omega)}^{2}+\left\|\hat{\phi}_{t}\right\|_{H_{\Gamma_{0}}^{1}(\Omega)}^{2}\right] d t \\
\leq & C_{T} \int_{0}^{T}\|\| \widehat{\phi}_{t} \mid \|_{L^{2}\left(\Gamma_{1}\right)}^{2} d t \\
& +C \sum_{i=1}^{2}\left\|\left[\hat{\phi}\left(s_{i}\right), \widehat{\phi}_{t}\left(s_{i}\right)\right]\right\|_{H_{\Gamma_{0}}^{2}(\Omega) \times H_{\Gamma_{0}}^{1}(\Omega)}^{2},
\end{aligned}
$$

where $s_{1}=T-\epsilon_{0}$, and $s_{2}=\epsilon_{0}$. 
Step 4. A standard energy argument shows that the heat component $\widehat{\psi}$ satisfies the estimate

$$
\begin{aligned}
\int_{0}^{T}\|\hat{\psi}\|_{H^{1}(\Omega)}^{2} d t \leq & C\left(\int_{0}^{T}\left[\left\|\frac{\partial \widehat{\phi}_{t}}{\partial \nu}\right\|_{L^{2}\left(\Gamma_{1}\right)}^{2}+\|\hat{\phi}\|_{H_{\Gamma_{0}}^{2}(\Omega)}^{2}\right] d t\right. \\
& \left.+\int_{0}^{T}\left\|\hat{\phi}_{t}\right\|_{H_{\Gamma_{0}}^{1}(\Omega)}^{2} d t\right)+ \text { I.o.t. }\left(\widehat{\phi}, \widehat{\phi}_{t}, \widehat{\psi}\right) .
\end{aligned}
$$

This inequality, in combination with Lemmas 4 and 5, eventually give (again the full details are in [4]),

Lemma 6 For $T>0$ large enough, the solution $\left[\widehat{\phi}, \widehat{\phi}_{t}, \widehat{\psi}\right]$ of (2.4) satisfies the following estimate:

$$
\begin{aligned}
& \int_{0}^{T}\left[\|\hat{\phi}\|_{H_{\Gamma_{0}}^{2}(\Omega)}^{2}+\left\|\hat{\phi}_{t}\right\|_{H_{\Gamma_{0}}^{1}(\Omega)}^{2}+\|\hat{\psi}\|_{H^{1}(\Omega)}^{2}\right] d t \\
+ & \left\|\left[\widehat{\phi}(T), \widehat{\phi}_{t}(T)\right]\right\|_{H_{\Gamma_{0}}^{2}(\Omega) \times H_{\Gamma_{0}}^{1}(\Omega)}^{2} \\
\leq & C_{T} \int_{0}^{T}\|\| \widehat{\phi}_{t} \mid \|_{L^{2}\left(\Gamma_{1}\right)}^{2} d t+\text { l.o.t. }\left(\widehat{\phi}, \widehat{\phi}_{t}, \widehat{\psi}\right) .
\end{aligned}
$$

Step 5. Note that in the inequality (2.6), there is no boundary trace term $\left.\psi\right|_{\Gamma_{2}}$, reflecting the contribution of the control $u_{3}$; the observability estimate (2.6) is independent of thermal control. However, to remove the corrupting lower order terms in this estimate so as to have the desired inequality (2.1), the thermal control now comes directly into play. Indeed, a compactnessuniqueness argument is now to be employed, with this argument making critical use of the Holmgren's-type uniqueness result derived in [7] for overdetermined (in both the mechanical and thermal variables) thermoelastic systems. The correct use of this uniqueness theorem necessitates the appearance of the thermal control $u_{3}$. With such control in place, we then have

Lemma 7 For $T>0$ large enough, the existence of the inequality (2.6) implies that there exists a $C_{T}$ such that the following estimate holds true:

$$
\text { I.o.t. }\left(\hat{\phi}, \widehat{\phi}_{t}, \hat{\psi}\right) \leq C_{T}\left(\int_{0}^{T}\left\|\left|\nabla \widehat{\phi}_{t}\right|\right\|_{L^{2}\left(\Gamma_{1}\right)}^{2} d t+\|\hat{\psi}\|_{\left[H^{s}\left(\Sigma_{2, T}\right)\right]^{\prime}}^{2}\right) \text {. }
$$

The inequalities (2.6) and (2.7), and the transformation $\widehat{\phi}(t)=e^{-\xi t} \phi(t)$ and $\widehat{\psi}(t)=e^{-\xi t} \psi(t)$ give the desired inequality (2.1), thereby completing the proof of Theorem 3. By the remarks made at the beginning of this section, with this partial exact controllability result in hand, the exact-approximate controllability statement Theorem 1 follows. 


\section{References}

[1] G. Avalos and I. Lasiecka, "Exponential stability of a free thermoelastic system without mechanical dissipation", SIAM Journal Math. Anal., Vol. 29, No. 1, (January 1998), pp. 155-182.

[2] G. Avalos and I. Lasiecka, "Exponential stability of a thermoelastic system without mechanical dissipation", Rendiconti Di Instituto Di Matematica Dell'Universitá di Trieste, Supp. Vol. XXVIII, 1-28 (1997).

[3] G. Avalos, "Exact controllability of a thermoelastic system with control in the thermal component only", IMA Preprint Series \#1532 (University of Minnesota), and submitted to Advances in Differential Equations.

[4] G. Avalos and I. Lasiecka, "Boundary controllability of thermoelastic plates with free boundary conditions", IMA Preprint Series \#1567 (University of Minnesota).

[5] L. de Teresa and E. Zuazua, "Controllability for the linear system of thermoelastic plates", Advances in Differential Equations, Vol. 1, Number 3 (1996), pp. 369-402.

[6] S. Hansen and B. Zhang, "Boundary control of a linear thermoelastic beam", J. Math. Anal. Appl., 210, (1997), 182-205.

[7] V. Isakov, "On the uniqueness of the continuation for a thermoelasticity system", preprint (1998).

[8] J. Lagnese, Boundary stabilization of thin plates, SIAM Stud. Appl. Math., 10 (1989).

[9] J. Lagnese, "The reachability problem for thermoelastic plates", Arch. Rational Mech. Anal., 112 (1990), pp. 223-267.

[10] I. Lasiecka and R. Triggiani, "Exact controllability of the wave equation with Neumann boundary control", Appl. Math. Optim., 19 (1989), 243-290.

[11] I. Lasiecka and R. Triggiani, "Sharp trace estimates of solutions to Kirchoff and Euler-Bernoulli equations", Appl. Math. Optim., 28 (1993), 277-306

[12] I. Lasiecka and R. Triggiani, "Structural decomposition of thermoelastic semigroups with rotational forces", to appear in Semigroup Forum.

[13] G. Lebeau and E. Zuazua, "Null-controllability of a system of linear thermoelasticity", Arch. Rational Mech., Vol. 141 (1998), 297-329.

[14] W. Liu, "Partial exact controllability and exponential stability in higherdimensional linear thermoelasticity", ESAIM: Control, Optimisation and Calculus of Variations, Vol. 3 (1998), 23-48.

[15] W. Liu, "Erratum on partial exact controllability and exponential stability in higher-dimensional linear thermoelasticity", to appear.

[16] D. Russell, "Controllability and stabilizability theory for linear partial differential equations: recent progress and open questions, SIAM Review, vol. 20, No. 4 (1978), 639-739. 